\title{
BMJ Global Health How to organise travel restrictions in the new future: lessons from the COVID-19 response in Hong Kong and Singapore
}

\author{
Daoyuan Lai (D) , ${ }^{1}$ Yuxi Cai (D) , ${ }^{1}$ Tsai Hor Chan (D) , ${ }^{1}$ Dailin Gan (D) , \\ Amber N Hurson (D) , ${ }^{3}$ Yan Dora Zhang (D) ${ }^{1,4}$
}

To cite: Lai D, Cai Y, Chan TH, et al. How to organise travel restrictions in the new future: lessons from the COVID-19 response in Hong Kong and Singapore. BMJ Global Health 2022;7:e006975. doi:10.1136/ bmjgh-2021-006975

Handling editor Seye Abimbola

Received 21 July 2021

Accepted 1 February 2022

A) Check for updates

(C) Author(s) (or their employer(s)) 2022. Re-use permitted under CC BY-NC. No commercial re-use. See rights and permissions. Published by BMJ.

${ }^{1}$ Department of Statistics and Actuarial Science, The University of Hong Kong, Hong Kong SAR, People's Republic of China ${ }^{2}$ Department of Applied and Computational Mathematics and Statistics, University of Notre Dame, Notre Dame, Indiana, USA

${ }^{3}$ Division of Cancer Epidemiology and Genetics, National Cancer Institute, Bethesda, Maryland, USA

${ }^{4}$ Centre for PanorOmic Sciences, Li Ka Shing Faculty of Medicine, The University of Hong Kong, Hong Kong SAR, People's Republic of China

Correspondence to Dr Yan Dora Zhang; doraz@hku.hk

\section{ABSTRACT}

It has been nearly 2 years since the first case of COVID-19 was reported. Governments worldwide have introduced numerous non-pharmaceutical interventions (NPIs) to combat this disease. Many of these NPIs were designed in response to initial outbreaks but are unsustainable in the long term. Governments are exploring how to adjust their current NPIs to resume normal activities while effectively protecting their population. As one of the most controversial NPIs, the implementation of travel restrictions varies across regions. Some governments have abandoned their previous travel restrictions because of the induced costs to society and on the economy. Other areas, including Hong Kong (Special Administrative Region of China) and Singapore, continue employing these NPIs as a long-term disease prevention tactic. However, the multidimensional impacts of travel restrictions require careful consideration of how to apply restrictions more appropriately. We have proposed an adapted framework to examine Hong Kong and Singapore's travel restrictions. We aimed to study these two regions' experiences in balancing disease control efforts with easing the burden on lives and livelihoods. Based on the experiences of Hong Kong and Singapore, we have outlined six policy recommendations to serve as the cornerstone for future research and policy practices.

\section{INTRODUCTION}

Worldwide, there is an ongoing struggle against the COVID-19 pandemic. Although the COVID-19 vaccine brings hope for ending this pandemic, some unsolved problems indicate that it may not be the silver bullet. ${ }^{12}$ Furthermore, experience from history suggests that vaccination alone is not sufficient to control a pandemic single-handedly and promptly. ${ }^{3}$ The global pandemic response should be supported by a combination of powerful non-pharmaceutical interventions (NPIs). Travel restrictions have been one of the most commonly used NPIs during the outbreak, proving their effectiveness in containing

\section{Summary box}

- Many studies showed that travel restrictions have successfully delayed the COVID-19 epidemic progression in the initial stage; however, investigating their effectiveness in the later phase becomes challenging as the global pandemic situation is rapidly evolving.

- Currently, many regions have eased or abandoned their original travel restrictions due to the induced social and economic costs; meanwhile, some areas still maintain tight restrictions on travel. These disparate responses imply the absence of an agreed methodology in organising these restrictions.

- This study summarised Hong Kong and Singapore's experience in using travel restrictions to protect public health in the COVID-19 pandemic and how they minimise the adverse impacts of these restrictions.

- This study outlined six policy prescriptions on how to manage COVID-19-related travel restrictions in the new normal.

- Our findings suggest that travel restrictions are essential COVID-19 prevention strategies, but they should not be the stumbling block for necessary international communications.

the disease in 2020 without acute economic fallout. ${ }^{4}$ A study in Nordic regions showed that closing the international border not only defended the local community from the COVID-19 but also supported the economic growth in $2020 .^{5}$ Nonetheless, many wellknown travel restrictions, such as complete border closure, were initially designed to buy time for understanding the epidemiological characteristics of this novel disease. ${ }^{4}$ These restrictions are often extreme and bring severe socioeconomic costs, making them unsustainable in the long term. For example, after the local epidemic situations had been controlled, many Asian-Pacific regions continued to suspend international travel to 
prevent the importation of SARS-CoV-2. Although these radical restrictions were the safest response at the time to the enormous uncertainties surrounding this novel disease, they deeply affected emergency and humanitarian actions, the movement of essential workers and the transportation of necessary goods. It will be beneficial for policy makers who have implemented these travel restrictions as part of a long-term response plan to consider easing the restrictions.

At the same time, many governments have already eased their original travel-related measures and have allowed non-essential international travel to resume. Amid the reasonable desire of returning to normalcy, however, it has become clear that easing travel restrictions increases the risk of virus importation and complexifies the disease transmission chain. Because many countries are not sufficiently protected by the vaccine-induced or infectioninduced immunity and lack an abundant health system capacity, the importation of novel variants of SARS-CoV-2 will still present a high public health risk. We argue that although some of the current travel restrictions should be eased, such adjustments can be enforced in a tailored fashion based on defined guidelines. These efforts can include conducting risk assessment systematically and regularly, improving quarantine arrangements, adopting extensive contact tracing, increasing COVID-19 testing frequency, tightening quarantine exemption arrangements and imposing punishments that enforce policy compliance.

\section{Concerns regarding COVID-19 vaccines}

Concerns about COVID-19 vaccines persist, as evidence has shown that some novel variants of SARS-CoV-2 are more resistant to the existing vaccines. For instance, research suggested that the emerging omicron (B.1.1.529) variant of SARS-CoV-2 can threaten the efficacy of current COVID-19 vaccines. ${ }^{6}$ Apart from this, the profound vaccine inequality is impairing the vaccine effectiveness, ${ }^{7}$ as many underdeveloped areas still suffer from inadequate COVID-19 vaccine supply. The lack of COVID-19 vaccines has significantly impeded these regions' pandemic control efforts, making them persistent hot spots of COVID-19 transmission. These regions tend to incubate novel variants of SARS-CoV-2, including delta (B.1.167.2) and omicron, which can be transmitted more easily and evade the existing immunity. ${ }^{8}$

To solve the vaccine inequality, worldwide governments have established many programmes, including the COVID-19 Vaccine Global Access (COVAX) programme to promote the global allocation of the COVID-19 vaccine. ${ }^{9}$ These programmes, however, are surrounded by many worrying uncertainties. A significant concern is that many high-income regions have bypassed the COVAX programme and have secured their vaccines directly from the developers. Although these rich countries only take up $16 \%$ of the global population, they have occupied $70 \%$ of the available COVID-19 vaccine doses. ${ }^{9}$ The severe vaccine inequality showed that many rich countries place their populations' vaccination process greatly ahead of the inoculation of vulnerable populations of underdeveloped countries. These policy makers should understand that solving the vaccine inequality is the best way to not only protect the people in low-income countries but also reduce the chance of emerging novel variants that can evade the established immunity for everyone. $^{10}$

\section{Effectiveness of travel restrictions}

Many studies showed that timely implementation of travel restrictions in the early stage of the pandemic likely slowed disease transmission. ${ }^{11}{ }^{12}$ Hong Kong banned flights from the UK 1 day after increased transmission of the Alpha variant (B.1.1.7) of SARS-CoV-2 was reported in the UK. ${ }^{13}$ Later, this variant was absent from Hong Kong's local population for almost half a year. ${ }^{14}$ At the same time, the USA strengthened cross-border regulations 8 days after the UK's report. ${ }^{15}$ Unfortunately, the Alpha variant was still detected in the US population before the travel restrictions came into effect. ${ }^{16}$ Indeed, policy implementation is haphazard, and thus early implementation may have minimal impacts on the number of people infected with novel variants of SARS-CoV-2. Nonetheless, Hong Kong and the USA's distinct epidemiological outcomes support the necessity of adopting timely, strict and targeted travel restrictions. Grépin et $a l^{11}$ argued that the early travel measures (eg, travel ban) implemented in Wuhan may have effectively shaped transmission within China, and additional travel measures such as suspending flights from or to China may have significantly reduced the internationally exported cases. Chinazzi et al ${ }^{12}$ estimated that Wuhan's travel measures reduced $77 \%$ of imported cases.

After the initial stage of the pandemic, travel restrictions have shown limited effectiveness in countries with high SARS-CoV-2 circulation because their local epidemic is driven by local rather than imported COVID-19 cases. ${ }^{17}$ However, travel restrictions are still essential for regions with low local COVID-19 transmission rates or many international arrivals. ${ }^{17}$ This is exemplified by the experiences of Mainland China, which maintained low local COVID-19 incidence rates through intensive border restrictions for most of $2020 .^{17}$

\section{Uncertainties of travel restrictions}

Although effective, travel restrictions can bring negative consequences in society, the economy, the global supply chain and more. ${ }^{11} 1819$ This is illustrated by the experiences of Hong Kong and Singapore, as the tourism industries of both regions were severely affected in 2020 (online supplemental figure 1). The cargo volume of Hong Kong's feeder sector had a $40 \%$ reduction in the first quarter of 2020 after the government tightened the import and export policies. ${ }^{20}$ Travel restrictions also pose considerable challenges to vulnerable groups. Lui et $a l^{21}$ showed that the strengthened travel restrictions lead to tremendous physical, mental and economic difficulties 
for Hong Kong's foreign domestic workers. Further, there is limited research establishing a widely accepted methodology to model the travel-related public health impacts in the COVID-19 context. ${ }^{22}$

The WHO initially recommended against placing any restrictions on travel and trade, based on available historical information and the principles of the International Health Regulations (2005). ${ }^{18}$ Later in the pandemic, however, it became clear that experiences from the previous pandemics were insufficient for decision-makers to control SARS-CoV-2 in an interdependent world. ${ }^{22}$ Therefore, the WHO now suggests countries to maintain basic risk mitigation measures related to international travel. These measures include introducing environmental methods, such as crowd control and hand hygiene measures, at the entry point or on conveyances. Furthermore, for those countries using supplementary measures, including adopting COVID-19 testing and quarantine as an entry condition, the WHO suggests guiding the implementation by a systematic risk assessment. ${ }^{23}$

\section{The stringency-flexibility trade-off of travel restrictions}

Governments generally implement travel restrictions with multiple health and non-health policy goals. According to Lee $e t a l,{ }^{24}$ these goals are often competing, with corresponding trade-offs. This can be contextualised as the stringency-flexibility trade-off of travel restrictions: on one hand, stringent travel restrictions certainly mitigate the importation and transmission risk of COVID-19 but also bring a myriad of adverse outcomes in society and the economy; on the other hand, relaxed travel restrictions may revitalise international travel while leaving the local population at risk. However, protecting public health and maintaining economic growth are not entirely conflicting. A recent study in Nordic countries showed that poorer public health outcomes are significantly associated with worse economic performance. ${ }^{5}$ After completely closing the national borders in 2020, Nordic countries not only reduced the number of COVID-19 infections but also revived their economies. It is noteworthy that Nordic regions made the achievement before the start of the global COVID-19 vaccination campaign, and thus this success may change in today's situation.

\section{RECOMMENDATIONS FOR IMPLEMENTING TRAVEL RESTRICTIONS}

Currently, more and more regions are adapting to the new normal. Social and economic activities are gradually resuming alongside looser containment measures to keep the infection rate manageable. Nevertheless, many people around the world still do not have access to COVID-19 tests, treatments and vaccines, with evolving variants adding further uncertainty on the trajectory of the pandemic. The growing pandemic complexity hampers evaluation of the impact of travel restrictions in this stage. To address this challenge, we first propose an adaptive framework to summarise travel restrictions based on the WHO guideline and the well-known Test-TraceIsolate-Quarantine strategy. ${ }^{25}$ Specifically, we categorise travel restrictions into six aspects: risk assessment of the global COVID-19 situation, quarantine arrangement for inbound travellers, COVID-19 testing requirement for inbound travellers, arrangement for exempted travellers and the penalty for breaching travel restrictions. Second, we present Hong Kong and Singapore as study cases. As two externally oriented economies, both regions have gained valuable experiences in using travel restrictions to minimise the importation risk of COVID-19. Their experiences suggest a way to achieve disease prevention while responding to social and economic concerns. Notably, their successes depend on their geographic and socioeconomic nature. We use Hong Kong and Singapore's experiences to guide global policy makers on better managing the stringency-flexibility trade-off of travel restrictions in the future. We demonstrate the details of the proposed framework in the Table 1. Online supplemental table 1 summarises how Hong Kong and Singapore's policies changed during the study period, that is, from 1 September 2020 to 20 August 2021.

\section{Limitations}

Admittedly, the socioeconomic disparities between the Asian and Western regions call into question the generalisability of Hong Kong and Singapore's experiences. In addition, Hong Kong and Singapore have adequate health resources, making some of their strategies infeasible in areas with vulnerable medical systems. Regarding the geographical aspect, Hong Kong and Singapore's NPIs may not be directly applicable to those regions with vast land boundaries. Both regions are mainly composed of maritime boundaries, and thus travel restrictions can be highly efficient in decelerating the transmission. ${ }^{16}$

\section{Pandemic situations in Hong Kong and Singapore}

Both Hong Kong and Singapore are island regions with high income, dense populations and prosperous tourism industries. Outbreak waves were not synchronous in these regions. From September 2020 to January 2021, Singapore achieved almost zero domestic COVID-19 infections, while Hong Kong experienced a local outbreak from November 2020 to March 2021 before finally controlling the outbreak. While Hong Kong and Singapore have maintained a low or almost zero transmission rate in the first half of 2021, Singapore's local incidence rate started to arise at the end of our study period as the country began to pivot from the zero-COVID approach (eliminating COVID-19 from the local population) to the endemic approach (coexisting with COVID-19).

Nevertheless, Hong Kong and Singapore's daily infection rates have been significantly lower than some Western countries (online supplemental figure 2). As two well-known Asian financial centres, imported cases accounted for a large proportion of Hong Kong and Singapore's daily confirmed cases (online supplemental figure 3). Therefore, travel-related measures are a 
Table 1 Overview of the latest travel restrictions taken by the Hong Kong and Singapore governments as of 20 August 2021

\section{Hong Kong}

\begin{tabular}{|c|c|c|}
\hline \multirow[t]{2}{*}{$\begin{array}{l}\text { Risk } \\
\text { assessment }\end{array}$} & Classification & $\begin{array}{l}\text { High-risk (Group A), medium-risk (Group B), low-risk } \\
\text { regions (Group C) }\end{array}$ \\
\hline & Travel ban & $\begin{array}{l}\text { Only allow entry for: (1) vaccinated Hong Kong } \\
\text { residents from Group A places; (2) all Hong Kong } \\
\text { residents and vaccinated non-Hong Kong residents } \\
\text { from Group B places; (3) all travellers from Group C } \\
\text { places }\end{array}$ \\
\hline \multirow[t]{5}{*}{ Quarantine } & $\begin{array}{l}\text { Financial } \\
\text { responsibility for } \\
\text { travellers }\end{array}$ & All self-funded \\
\hline & Private transport & Yes \\
\hline & Visitor ban & Yes \\
\hline & Length of stay & $\begin{array}{l}\text { Group A: } 21 \text { days } \\
\text { Group B: } 21 \text { days (unvaccinated)/14 days (vaccinated) } \\
\text { Group C: } 14 \text { days (unvaccinated)/7 days (vaccinated) }\end{array}$ \\
\hline & Facility & Group A/B/C: designated hotels \\
\hline
\end{tabular}

Facility

\section{Singapore}

Category I, II, III and IV with risk increasing from I to IV

Deny/tighten the entry approvals of selected regions; established the VTL

All self-funded

Yes

Yes

Category I: 0 days

Category II: 7 days

Category III/IV: 14 days

Category I: Not required

Category II: Traveller's chosen accommodation Category III: Dedicated SHN facilities (if not fully vaccinated)/Home (if fully vaccinated) Category IV: Dedicated SHN facilities

Surveillance Location-sharing software, video calls, manual spot- Electronic monitoring device, physical spot-checks checks

$\begin{array}{clll}\text { Contact tracing } & \text { Forward } & \text { Yes } & \text { Yes } \\ & \text { Backward } & \text { No } & \text { Yes }\end{array}$

COVID-19 Pre-departure test Negative PCR test result for all travellers within the testing certificate past 72 hours

On-arrival PCR test for all travellers

Before compulsory Group A: 6 PCR tests

quarantine ends Group B: 6 PCR tests (unvaccinated)/4 PCR tests (vaccinated)

Group C: 4 PCR tests (unvaccinated)/2 PCR tests (vaccinated)

After compulsory Group A: 1 PCR test

quarantine ends Group B: 2 PCR tests (vaccinated)

Group C: 4 PCR tests (vaccinated)/2 PCR tests (unvaccinated)

Financial ART: self-funded

responsibility for PCR: free

Yes

Yes

Category I/II: Not required

Category III/IV: Negative PCR test results within 72 hours

VTL: Negative PCR test result within 48 hours

PCR/ART test for all travellers

During SHN: 3 ART tests for travellers serving SHN at dedicated facilities

Exit SHN: 1 PCR test for travellers from Category II/III/ IV places

VTL: 2 PCR tests on days 3 and 7 of traveller's stay in Singapore

Self-funded travellers

\begin{tabular}{|c|c|c|c|}
\hline \multirow[t]{2}{*}{ Exemption } & Maritime workers & $\begin{array}{l}\text { Suspending crew change arrangement of Group A, } \\
\text { banning vessel crew members from going ashore, } \\
\text { pre-departure PCR test (within prior } 48 \text { hours), on- } \\
\text { arrival PCR test, point-to-point transportation }\end{array}$ & $\begin{array}{l}\text { 1. Locally based workers: comprehensive } \\
\text { vaccination, } 7 \text { day RRT, frequent PCR test, } \\
\text { administer PCR tests to shore-based workers } \\
\text { boarding ships, and a segregation arrangement } \\
\text { afterward, enhanced safety management measures } \\
\text { 2. Inbound workers: additional stay and testing } \\
\text { regime at dedicated facilities after 14-day SHN }\end{array}$ \\
\hline & Aircrew & $\begin{array}{l}\text { Pre-departure (within prior } 48 \text { hours) and on-arrival } \\
\text { PCR tests, point-to-point transportation, hotel } \\
\text { isolation until next duty flight, shortened isolation } \\
\text { period for local-based aircrew, an additional PCR } \\
\text { test before leaving isolation or before duty flight's } \\
\text { departure }\end{array}$ & $\begin{array}{l}\text { 1. Arriving aircrew: PCR test, } 7 \text { day RRT and } \\
\text { additional ART test for high-risk aircrews, } \\
\text { vaccination of aviation workers } \\
\text { 2. Layover aircrew: } 2 \text { PCR tests and self-isolation } \\
\text { before receiving a negative PCR test result }\end{array}$ \\
\hline Penalty & & $\begin{array}{l}\text { Up to HKD } 25000 \text { fine and/or up to } 6 \text { month } \\
\text { imprisonment }\end{array}$ & $\begin{array}{l}\text { Up to SGD } 10000 \text { fine, removal of PR's/SC's permit/ } \\
\text { pass, up to } 6 \text { month jail time }\end{array}$ \\
\hline
\end{tabular}

The detailed policy changes from 1 September 2020 to 20 August 2021 can be accessed in online supplemental table 1.

ART, antigen rapid test; HKD, Hong Kong Dollar; PR, permanent resident; RRT, rostered routine COVID-19 testing; SC, Singapore citizen; SGD, Singapore Dollar; SHN, stay home notice; VTL, Vaccine Travel Lane. 
significant component of their disease prevention tactics. As putting restrictions on travel also brings some undesirable consequences, these two regions are exploring how to apply travel restrictions more appropriately. Their experiences can aid policy makers in organising more discerning travel restrictions.

\section{Recommendation 1: systematic and regular risk assessment}

Public health risk assessment of inbound travellers' departure regions can provide the destination government with guidance for stratifying risk mitigation measures. For example, travellers from high-risk areas should be subject to stricter restrictions. In some extreme scenarios (eg, when facing novel variants that may break the established immunity), governments may temporarily shut down the borders (ie, implement travel bans) to prevent their health systems from becoming overwhelmed, with specific exemptions to protect necessary import and export travel. These precautionary actions, however, may lead to enormous economic and humanitarian hardship. ${ }^{24}$ Alternatively, governments can relax regulations for travellers from regions with a low local incidence rate or a high vaccination rate. For example, the WHO suggested exempting fully vaccinated travellers or persons with a valid certificate of previous SARS-CoV-2 infection from the COVID-19 test and quarantine requirements. ${ }^{23}$ However, relaxing or exempting disease prevention measures according to the traveller's immunisation status may introduce additional risks, as some existing immune-escape variants can break the established immunity and cause reinfection. ${ }^{23}$ Notwithstanding the side effects, a targeted strategy can better allocate scarce local health resources and minimise the negative impacts of implementing restrictions on international travel. ${ }^{26}$

The WHO suggested multiple critical factors to be considered for a well-built risk assessment list, including the local epidemic situation, the travel volume of the departure and arrival regions and the health system capacity of the arrival region. ${ }^{25}$ Initially, Hong Kong and Singapore classified regions as simply high-risk or low-risk, but their classifications have evolved over time. Recently, Hong Kong specified Groups A, B and C as high-risk, medium-risk and low-risk regions, respectively. Group A also includes selected countries for enhanced surveillance on the omicron variant. In August 2021, Singapore instituted a risk classification system with Categories I/II/III/IV (with risk increasing from Category I to IV). Beyond this classification system, Singapore also established the Vaccine Travel Lane (VTL) programme for travellers from specified regions to explore applying reduced border measures based on the travellers' vaccination status. ${ }^{27} 28$

Policy makers should regularly and systematically update their risk categories and proactively adjust the travel restrictions to ensure policy efficacy. The example of the Alpha variant in the Introduction demonstrates that some regions have underestimated the prevalence of the novel SARS-CoV-2 variants and have failed to establish travel regulations promptly. ${ }^{16}$ Some countries with low COVID-19 transmission closed their border to address this problem. However, the WHO warned that a blanket travel ban may not be evidence-based and may cause a severe bias towards countries actively sharing epidemiological and viral sequencing findings. ${ }^{23}$

\section{Recommendation 2: robust and centralised quarantine}

Implementing compulsory quarantine for inbound travellers is essential to detect potential COVID-19 carriers before they enter the local community. ${ }^{29}$ The measure can be classified into centralised quarantine and noncentralised quarantine. The former concentrates inbound travellers in the sites managed by the local or national jurisdictions, while the latter allows inbound travellers to choose suitable quarantine facilities such as their home. Hong Kong and Singapore mainly practiced centralised quarantine before August 2021. While Hong Kong is still maintaining this quarantine mode for all travellers from regions outside China, Singapore's primary quarantine mode has changed to a non-centralised one since adopting an endemic approach in August 2021. Currently, Singapore allows travellers to serve their stayhome notice (SHN) at their chosen accommodation, including their place of residence. Only Category IV travellers need to serve their SHN at a dedicated facility.

Considering the high-risk nature of novel SARS-CoV-2 variants, implementing centralised quarantine should be the primary choice. Hong Kong's regulations on quarantine hotels include: (1) fulfilling the standard of infection control, (2) banning local visitors from physically contacting inbound travellers, (3) implementing enhanced disinfection arrangements and (4) conducting routine COVID-19 tests for relevant quarantine hotel staff. ${ }^{30}$ Singapore's designated SHN facilities have similar requirements as Hong Kong. These regulations can be a guideline for building a robust centralised quarantine system.

Quarantine regulations must be well established. Otherwise, improper organisation of centralised quarantine facilities may lead to a severe outcome, especially in a low COVID-19 tolerance region (eg, a region with zero active cases or sporadic small outbreaks). Using phylogenomic analysis methods, Chan $e t a l^{31}$ concluded that Hong Kong's November 2020 resurgence was likely due to imported COVID-19 cases. A possible explanation is that there was a transmission from the quarantine hotel to the local community via direct contact with hotel personnel or indirect contact with inanimate objects. ${ }^{31}$ To address this concern, the Hong Kong government (1) banned inbound travellers from taking public transportation to the quarantine location, (2) suspended visitation to quarantine rooms from 18 December 2020 and (3) centralised inbound travellers from outside China in the designated hotel from 25 December $2020 .^{30}$

Regardless of the quarantine mode, surveillance of quarantined individuals is indispensable for building a robust quarantine system. Hong Kong and Singapore's 
relevant local authorities use spot-checks to ensure individual compliance with the quarantine measures. Moreover, digital tools can be applied to compensate for the shortage of disease control personnel. Hong Kong and Singapore require individuals under compulsory quarantine to wear an electronic monitoring device and download a mobile application. ${ }^{32} 33$

Despite the public health advantages, compulsory and centralised quarantine requirements may bring a series of negative externalities, including hindering the transportation of essential supplies, disrupting travel and trade, and reducing workforce mobility. ${ }^{23}$ Furthermore, some research has shown the potential adverse psychological effects of this NPI, including the association between longer quarantine duration and worse mental health. ${ }^{34}$

\section{Recommendation 3: extensive contact tracing}

A comprehensive and sophisticated contact tracing system helps identify the infection source of the local transmission chain and ultimately reveals potential defects in the border control system. It is essential to build a professional contact tracing team with health professionals. Digital tools are also vital to improve tracing efficiency. Singapore has developed two applications: TraceTogether (https://www.tracetogether.gov.sg), which uses mobile phone Bluetooth signal to pinpoint potential close contacts; and SafeEntry (https:/ / www.safeentry.gov. $\mathrm{sg}$ ), which uses QR codes to record users' travel history to public spaces. Hong Kong's LeaveHomeSafe (https:// www.leavehomesafe.gov.hk/en/) application functions similarly to SafeEntry.

Bidirectional contact tracing can improve disease control performance, as it combines forward tracing (tracing to the disease transmission source) and backward tracing (tracing from the disease transmission source). ${ }^{35}$ While bidirectional contact tracing has been well used in Singapore; Hong Kong only conducts forward tracing. Hong Kong lacks a city-wide digital contact tracing platform, with epidemic investigation relying heavily on the self-reported travel history of confirmed cases. This method can hamper the efficiency of contact tracing efforts as it cannot identify the transmission chain promptly.

Contact tracing inevitably brings privacy concerns. Some residents or travellers may be reluctant to provide their digital information for contract tracing. ${ }^{36}$ To relieve public hesitancy, the Hong Kong government has made several promises when promoting LeaveHomeSafe, including (1) storing visit records exclusively in the user's cell phone, (2) automatically erasing the digital footprints after 31 days and (3) conducting privacy assessments via reliable third-party organisations. ${ }^{37} \mathrm{Simi}$ larly, Singapore's TraceTogether only retains data for 21 days, and only has access to close contacts' information. ${ }^{38}$ However, it was found that the Singapore police may use the data for criminal investigations, leading to some public outcry. ${ }^{39}$

\section{Recommendation 4: frequent COVID-19 tests}

Thorough COVID-19 testing for inbound travellers can help detect those potential high-risk SARS-CoV-2 carriers. Routine pre-departure COVID-19 testing can filter out infected cases before their departure. For example, Kiang et $a l^{40}$ showed its effectiveness in reducing infection risk during airline travel. Hong Kong required inbound travellers to submit a self-funded negative COVID-19 PCR test certificate issued by a recognised institution within 72 hours before their entry. Singapore also has a similar requirement for high-risk travellers (ie, travellers from Category III/IV places), while Singapore's VTL requires the test must be conducted within 48 hours. ${ }^{27}$ Nevertheless, a negative pre-departure COVID-19 test result is not perfectly reliable, as some travellers may have an undetectable viral load when they undergo the test. ${ }^{41}$

To make up for the limitations of pre-departure testing, compulsory COVID-19 tests are still essential for all inbound travellers on their arrival and during the quarantine. Hong Kong has recently increased its COVID-19 test frequency as the damaging new variants of SARSCoV-2 are challenging the border control efforts. The government requires at least four PCR tests during the 21-day quarantine of inbound travellers from Group A or Group B regions. Like Hong Kong, Singapore requires all inbound travellers to undergo on-arrival COVID-19 testing. As of 19 August 2021, travellers from Category II/III/IV regions are required to conduct additional COVID-19 testing before leaving the quarantine facilities. Travellers within Singapore's VTL programme must undergo two PCR tests after their arrival. ${ }^{27}$

Certain flexibilities in the COVID-19 testing requirements are worth exploring. First, policy makers may consider amending the testing arrangement as a means to ease quarantine. Hong Kong has recently shortened the compulsory quarantine period for people from lowrisk regions or who have been fully vaccinated. To reduce the potential risk of this relaxation, the government has increased the frequency of compulsory PCR tests during and after the mandatory quarantine period. ${ }^{27}$ Singapore's on-arrival test requirement was temporally suspended for inbound travellers from Category II/III/IV regions in October 2021. However, this requirement has recently been readopted as a precautionary measure towards the scientific uncertainties of the omicron variant. ${ }^{42}$ Second, accepting various test measures (eg, antigen rapid test) apart from the usual PCR test may benefit more travellers, including those who have recovered from a previous infection. Since 7 August 2021, Hong Kong has offered a self-paid on-arrival serology antibody test at the Hong Kong International Airport for vaccinated travellers from Group B regions. The compulsory quarantine period for these travellers could be shortened to 7 days if they received a positive test result. ${ }^{43}$ However, on 20 August 2021, the government shelved this arrangement in light of the evolving global pandemic situation and the latest suggestions from experts. ${ }^{44}$ Singapore has started to use 
the antigen rapid test for on-arrival COVID-19 test from 19 August $2021 .^{27}$

The enhanced COVID-19 test requirements also bring some undesired consequences. They inevitably encourage illegal actions such as forging test certificates, and impose an invisible burden on travellers who cannot obtain a valid test certificate or bear the cost of a COVID-19 test. ${ }^{22}$ While Hong Kong pays for the COVID-19 testing cost, Singapore's inbound travellers must cover the cost of their tests. Regardless, mandatory COVID-19 testing requirements are not sufficient to defend the spread of SARS-CoV-2. Research has shown that rigorous PCR testing may still miss many potential SARS-CoV-2 carriers. ${ }^{45}$ Therefore, COVID-19 testing requirements must be used in combination with additional measures to control disease transmission.

\section{Recommendation 5: limited quarantine exemptions}

It is necessary to relax the quarantine measures for specific travellers (ie, the exemption cluster), including aircrew, maritime workers and other people with exceptional reasons (eg, diplomatic issues), as these groups are essential for the normal functioning of society. In Hong Kong, $93 \%$ of the exempted persons are aircrew, $5 \%$ are maritime workers and $2 \%$ are consular and diplomatic officers. ${ }^{46}$ However, as these people have complicated travel histories and have remained in a closed high-risk environment for extended periods, careful management is needed to mitigate risk.

Hong Kong has paid greatly for underestimating the risk of the exemption cluster. For example, To $e t a l^{77}$ showed that Hong Kong's infection resurgence in July 2020 may be associated with exempted maritime crew or aircrew. To address the rising concern regarding the exemption cluster, Hong Kong cancelled vessels' crew change arrangement from 29 July $2020 .^{48}$ Additionally, starting from 21 November 2020, the government further limited all airline worker's movement in the local community, tightened their self-isolation quarantine requirements and enhanced the COVID-19 testing requirement. ${ }^{46}$ Initially, Singapore did not require aircrew to serve the SHN if they had taken stringent precautions. Later (as of 20 December 2020), the government increased the PCR test frequency for aircrew and required them to stay selfisolated until receiving a negative test result. ${ }^{49}$

The experiences of Hong Kong and Singapore highlight some critical insights for managing the exemption cluster. First, a closed-loop arrangement for exemption clusters can reduce the risk of virus importation. For example, Singapore's Changi Airport has assigned a particular pier for flights from high-risk regions. ${ }^{50}$ Second, a shortened quarantine period and enhanced surveillance must be conducted simultaneously for the exemption cluster. ${ }^{51}$ Before May 2021, Taiwan allowed cargo aircrews to leave the quarantine location after the third day of the quarantine without any compulsory COVID-19 test requirements. ${ }^{52}$ Unfortunately, the subsequent outbreak indicates that such a relaxed measure can introduce extra risk, which is exceptionally significant in a community with low COVID-19 incidence rates. Third, vaccination requirements for the exemption cluster can better protect this highly risky and vulnerable group. Singapore had already vaccinated more than $90 \%$ of its aviation workers by January $2021 .^{50}$

It is worth mentioning that tightening the quarantine exemption requirement brings many costs, including impeding the travel of essential workers (eg, healthcare workers and emergency responders), interrupting the transport of essential supplies (eg, food, fuel and medicines) and hampering the humanitarian actions (eg, emergency evacuation). ${ }^{23}$

\section{Recommendation 6: enforcing penalty for breaching disease prevention regulations}

Inbound travellers' adherence to the regulations is indispensable to maintain the effectiveness of travel restrictions. Many regions, including Hong Kong and Singapore, have introduced legal penalties to improve public compliance. Individuals who violate Hong Kong's compulsory quarantine orders are subject to a maximum fine of 25000 Hong Kong Dollar (HKD) and up to 6 months imprisonment. ${ }^{53}$ Apart from the maximum 10 000 Singapore Dollar fine and 6 month sentence, Singapore may also revoke the working permit of individuals who breach the SHN requirements. ${ }^{54}$ Notably, the desired regulatory outcomes are hard to achieve without adequate enforcement. Hong Kong and Singapore have taken responsive actions. As of 17 December 2021, Hong Kong had convicted 205 individuals for breaching the quarantine requirement. Their sentences included up to 14 weeks' imprisonment and a maximum fine of 15 $000 \mathrm{HKD}^{55}$ Singapore recently fined 47 persons and suspended seven maritime companies' working licenses for breaching the COVID-19 prevention measures. ${ }^{56}$ However, further research is needed to clarify the effectiveness of these measures.

Apart from targeting individual travellers, governments can also introduce disease prevention regulations and penalties on international transport operators to motivate compliance and reduce administrative costs. Hong Kong has set up a circuit breaker mechanism for conveyance operators. They will face punishments if the relevant travellers on their flights violate travel restrictions. For example, all airlines' flights from one specific place will be banned from arriving in Hong Kong for 14 days when five or more passengers, among all civil aircraft from a particular area, are confirmed positive with the N501Y mutant strain within 7 days on the arrival. ${ }^{57}$ In Singapore, if a maritime worker fails to cooperate with disease prevention measures, the corresponding craft's or vessel's license will be suspended for 30 days. ${ }^{56}$

Enforcement of stringent penalties may be counterproductive. Worsnop ${ }^{58}$ argued that inbound travellers may refuse to report relevant information for fear of severe consequences. Furthermore, enforcement of these penalties surely raises many ethical concerns and may 
jeopardise the interests of passengers and the operations of conveyance companies.

However, given the high costs of re-introducing SARSCoV-2 and its new variants to regions with low risk tolerance, stringent penalties can motivate individuals and companies to comply with travel restrictions. Imposing legal penalties such as fines or imprisonment may effectively prevent travellers from breaking quarantine orders (eg, leaving the quarantine facility without permission or refusing to take the COVID-19 test). At the same time, legal measures applied to companies, such as circuit breaker measures, can reduce the number of imported cases effectively, as suggested by the experience of Mainland China. ${ }^{59}$ The measure can also motivate the conveyance operators to actively comply with the destination government's disease-prevention regulations. Meanwhile, some actions are necessary to reduce the costs of these penalties, including (1) fully involving and consulting travellers before making any changes to disease regulations, (2) clearly and concisely informing travellers about the details of related regulations and (3) assessing the effectiveness and potential impacts of these penalties through an evidence-based approach. ${ }^{26}$ Finally, countries with higher risk tolerance may consider these penalties as supplements to their current travel-related measures if necessary.

\section{DISCUSSION}

We have proposed an adapted framework to retrospectively review the travel restrictions implemented by Hong Kong and Singapore from 1 September 2020 to 20 August 2021. Their stringent travel restrictions have protected the health of their local population, but also inevitably introduced a heavy burden on lives and livelihoods. To address these adverse impacts, Hong Kong and Singapore have also explored how to adopt travel restrictions as an effective and adaptable response to the pandemic. Their experiences serve as a model that will be useful for global policy makers and researchers.

Furthermore, as we mentioned previously, striking a balance between stringency and flexibility is a fundamental challenge in policy implementation-especially when facing today's complex epidemic situation and the need for regular international communication. This dilemma calls for further research on an agreed-upon protocol for implementing travel restrictions. In the following discussion, we raise several points worthy of being further studied.

First, it remains to be seen how Hong Kong and Singapore will differ in their public health and economic outcomes as they move in different policy directions. While Hong Kong and Singapore both pursued a zeroCOVID strategy from September 2020 to August 2021, Singapore is transitioning to an endemic approach. Specifically, Singapore is increasingly resuming social, economic and travel activities after over $80 \%$ of their local population were vaccinated. However, this country has since experienced soaring COVID-19 infection rates, although hospitalisation and death rates have remained relatively low. ${ }^{60}$ Consideration of Hong Kong and Singapore's future public health and economic outcomes will inform a better way to ease the negative impacts of the zero-COVID approach when a region achieves a sufficiently high vaccination rate. The long-term sustainability of the current zero-COVID approach is worth discussing, as some regions sticking to this strategy still encounter local outbreaks from time to time. For example, Hong Kong has recently encountered small-scale outbreaks, despite the government implementing stringent travel restrictions and local disease elimination strategies since 2020.

Second, it is worth investigating how to use travellers' immunisation status as a criterion for easing travel restrictions. Many governments have begun to explore this approach. Singapore's VTL programme allows inbound quarantine-free travel for those who are fully inoculated with a COVID-19 vaccine listed in the WHO's Emergency Use Listing, hold valid vaccination proof and fulfil travel history requirements. ${ }^{28}$ The European Union has introduced a digital COVID-19 certificate for those who have been vaccinated, received a negative PCR test result, or recovered from COVID-19. However, whether such an immunity certificate is effective remains unclear. The WHO remains conservative about endorsing the use of COVID-19 certificates because there is limited scientific evidence showing that individuals with a previous infection and antibodies are not at risk of reinfection. ${ }^{25}$ There are also concerns about the inequalities that COVID-19 certificates may bring, as many underdeveloped regions still suffer from vaccine shortages and delays.

Third, there is a paucity of research on the interactions between coexistent travel restrictions and local control measures. Cross-border contact tracing is an example of such an interaction, where regions collaborate to conduct cross-sectional contact tracing after a case is identified as infected during cross-border travel. ${ }^{25}$ Hong Kong has organised an information-sharing mechanism with Guangdong Province of Mainland China to monitor the travel history of cross-boundary goods vehicle drivers, aiming to identify closed contacts of the infected drivers promptly. ${ }^{61}$ To address the concerns over the erosion of privacy in contact tracing, governments should value the protection of personal information and may adopt encrypted communication, as the WHO has recommended. ${ }^{25}$

\section{CONCLUSION}

Implementing travel restrictions is vital to prevent higher rates of SARS-CoV-2 transmission and more severe disease in local communities. The six proposed policy prescriptions may serve as the cornerstone for future research and policy practices. Given the ongoing nature of the current COVID-19 pandemic, we hope that global policy makers can use our suggestions to reduce the negative impacts 
of policy, balance the stringency-flexibility trade-off and protect population health safely and appropriately.

Twitter Daoyuan Lai @DaoyuanLai and Yan Dora Zhang @happy_dora1

Acknowledgements The authors gratefully acknowledge Eric Fu for his insightful suggestions and continuous encouragement and two anonymous reviewers who helped significantly strengthen the article.

Contributors DL and YDZ conceived the study. DL, YC, THC and DG collected the data. $\mathrm{DL}$ and $\mathrm{YC}$ conducted the systematic review search and data synthesis. $\mathrm{DL}$ and YDZ wrote the first draft of the manuscript. DL, YC, ANH and YDZ critically revised the manuscript. All authors have commented on the different versions of the manuscript and approved the final version. YDZ had full access to all the data in the study and had final responsibility for the decision to submit for publication.

Funding The authors have not declared a specific grant for this research from any funding agency in the public, commercial or not-for-profit sectors.

Competing interests None declared.

Patient consent for publication Not applicable.

Provenance and peer review Not commissioned; externally peer reviewed.

Data availability statement Data are available in a public, open access repository.

Supplemental material This content has been supplied by the author(s). It has not been vetted by BMJ Publishing Group Limited (BMJ) and may not have been peer-reviewed. Any opinions or recommendations discussed are solely those of the author(s) and are not endorsed by BMJ. BMJ disclaims all liability and responsibility arising from any reliance placed on the content. Where the content includes any translated material, BMJ does not warrant the accuracy and reliability of the translations (including but not limited to local regulations, clinical guidelines, terminology, drug names and drug dosages), and is not responsible for any error and/or omissions arising from translation and adaptation or otherwise.

Open access This is an open access article distributed in accordance with the Creative Commons Attribution Non Commercial (CC BY-NC 4.0) license, which permits others to distribute, remix, adapt, build upon this work non-commercially, and license their derivative works on different terms, provided the original work is properly cited, appropriate credit is given, any changes made indicated, and the use is non-commercial. See: http://creativecommons.org/licenses/by-nc/4.0/.

\section{ORCID iDs}

Daoyuan Lai http://orcid.org/0000-0002-1080-7110

Yuxi Cai http://orcid.org/0000-0003-4065-1193

Tsai Hor Chan http://orcid.org/0000-0002-3545-397X

Dailin Gan http://orcid.org/0000-0003-0535-1259

Amber N Hurson http://orcid.org/0000-0001-7831-6660

Yan Dora Zhang http://orcid.org/0000-0002-5302-3690

\section{REFERENCES}

1 Lopez Bernal J, Andrews N, Gower C, et al. Effectiveness of Covid-19 vaccines against the B.1.617.2 (delta) variant. N Engl $J$ Med Overseas Ed 2021;385:585-94.

2 Del Rio C, Malani P. COVID-19 in 2021-Continuing uncertainty. JAMA 2021;325:1389-90.

3 Oliu-Barton M, Pradelski BSR, Aghion P, et al. SARS-CoV-2 elimination, not mitigation, creates best outcomes for health, the economy, and civil liberties. Lancet 2021;397:2234-6.

4 De Foo C, Grépin KA, Cook AR, et al. Navigating from SARS-CoV-2 elimination to endemicity in Australia, Hong Kong, New Zealand, and Singapore. Lancet 2021;398:1547-51.

5 Gordon DV, Grafton RQ, Steinshamn SI. Cross-country effects and policy responses to COVID-19 in 2020: the Nordic countries. Econ Anal Policy 2021;71:198-210.

6 Liu L, Iketani S, Guo Y, et al. Striking antibody evasion manifested by the omicron variant of SARS-CoV-2. Nature 2021. doi:10.1038/ s41586-021-04388-0. [Epub ahead of print: 23 Dec 2021].

7 United Nations Office for the Coordination of Humanitarian Affairs. Africa faces looming health disaster as COVID-19 cases surge, delta variant spreads. Available: https://reliefweb.int/report/world/africafaces-looming-health-disaster-covid-19-cases-surge-delta-variantspreads

8 Tatar M, Shoorekchali JM, Faraji MR, et al. International COVID-19 vaccine inequality amid the pandemic: perpetuating a global crisis? J Glob Health 2021;11:03086.
9 Wouters OJ, Shadlen KC, Salcher-Konrad M, et al. Challenges in ensuring global access to COVID-19 vaccines: production, affordability, allocation, and deployment. Lancet 2021;397:1023-34.

10 Moore JP, Offit PA. SARS-CoV-2 vaccines and the growing threat of viral variants. JAMA 2021;325:821-2.

11 Grépin KA, Ho T-L, Liu Z, et al. Evidence of the effectiveness of travel-related measures during the early phase of the COVID-19 pandemic: a rapid systematic review. BMJ Glob Health 2021;6:e004537.

12 Chinazzi M, Davis JT, Ajelli M, et al. The effect of travel restrictions on the spread of the 2019 novel coronavirus (COVID-19) outbreak. Science 2020;368:395-400.

13 Hong Kong Government. Government strengthens restrictions to persons arriving at Hong Kong from United Kingdom. Available: https://www.info.gov.hk/gia/general/202012/22/P2020122200030. htm? fontSize $=1$

14 RTHK. Secondary school girl contracts UK Covid-19 variant. Available: https://news.rthk.hk/rthk/en/component/k2/159441820210605.htm

15 United States Department of State. FAQ: Travel Restrictions Related to New UK COVID Strain. Available: https://www.osac.gov/Country/ UnitedKingdom/Content/Detail/Report/51190251-2eb5-475d-a35c$1 \mathrm{a} 747166 \mathrm{fb} 9 \mathrm{c}$

16 Grubaugh ND, Hodcroft EB, Fauver JR, et al. Public health actions to control new SARS-CoV-2 variants. Cell 2021;184:1127-32.

17 Russell TW, Wu JT, Clifford S, et al. Effect of internationally imported cases on internal spread of COVID-19: a mathematical modelling study. Lancet Public Health 2021;6:e12-20.

18 Lee K, Worsnop CZ, Grépin KA, et al. Global coordination on crossborder travel and trade measures crucial to COVID-19 response. Lancet 2020;395:1593-5.

19 World Health Organization. Weekly operational update on COVID-19, 2020. Available: https://www.who.int/publications/m/item/weeklyupdate-on-covid-19-15-april-2020

20 The Chartered Institute of Logistics and Transport in Hong Kong. Impact of COVID-19 on Hong Kong's Logistics Industry. Available: https://www.ciltinternational.org/wp-content/uploads/2020/07/ Impact-of-COVID-19-on-Hong-Kongs-Logistics-Industry-20200713. $\mathrm{pdf}$

21 Lui ID, Vandan N, Davies SE, et al. "We also deserve help during the pandemic": The effect of the COVID-19 pandemic on foreign domestic workers in Hong Kong. J Migr Health 2021;3:100037.

22 Lee K. Restarting international travel will be messy but enabled by improved risk-based analyses. Lancet Reg Health West Pac 2021;13:100209.

23 World Health Organization. Technical considerations for implementing a risk-based approach to international travel in the context of COVID-19: interim guidance, 2021. Available: https:// www.who.int/publications/i/item/WHO-2019-nCoV-Risk-basedinternational-travel-2021.1

24 Lee K, Grépin KA, Worsnop C, et al. Managing borders during public health emergencies of international concern: a proposed typology of Cross-border health measures. Global Health 2021;17:1-19.

25 World Health Organization. Considerations for implementing a riskbased approach to international travel in the context of COVID-19. Available: https://www.who.int/publications/i/item/WHO-2019-nCoVRisk-based-international-travel-2020.1

26 World Health Organization. Considerations for implementing and adjusting public health and social measures in the context of COVID-19. Available: https://www.who.int/publications/i/item/ considerations-in-adjusting-public-health-and-social-measures-inthe-context-of-covid-19-interim-guidance

27 Singapore Ministry of Health. Next steps in our transition towards Covid resilience. Available: https://www.moh.gov.sg/newshighlights/details/next-steps-in-our-transition-towards-covidresilience

28 Singapore Immigration and Checkpoints Authority. Vaccinated travel Lane (VTL) overview. Available: https://safetravel.ica.gov.sg/vtl/ requirements-and-process

29 Han E, Tan MMJ, Turk E, et al. Lessons learnt from easing COVID-19 restrictions: an analysis of countries and regions in Asia Pacific and Europe. Lancet 2020;396:1525-34.

30 Hong Kong Government. Designated quarantine hotel scheme fully implemented today. Available: https://www.info.gov.hk/gia/general/ 202012/22/P2020122200839.htm?fontSize =1

31 Chan W-M, Ip JD, Chu AW-H, et al. Phylogenomic analysis of COVID-19 summer and winter outbreaks in Hong Kong: an observational study. Lancet Reg Health West Pac 2021;10:100130.

32 Hong Kong Government. LCQ11: application of technology in efforts to combat COVID-19 pandemic. Available: https://www.ogcio.gov. hk/en/news/press_releases/2020/06/pr_20200603.html 
33 Singapore Government. SHN electronic monitoring device. Available: https://safetravel.ica.gov.sg/health/shn-monitoring

34 Brooks SK, Webster RK, Smith LE, et al. The psychological impact of quarantine and how to reduce it: rapid review of the evidence. Lancet 2020;395:912-20.

35 Bradshaw WJ, Alley EC, Huggins JH, et al. Bidirectional contact tracing could dramatically improve COVID-19 control. Nat Commun 2021;12:232

36 Grande D, Mitra N, Marti XL, et al. Consumer views on using digital data for COVID-19 control in the United States. JAMA Netw Open 2021;4:e2110918-e18.

37 Hong Kong Government. LeaveHomeSafe homepage. Available: https://www.leavehomesafe.gov.hk/en/

38 Singapore Government. TraceTogether Homepage. Available: https://www.tracetogether.gov.sg/

39 Schaefer GO, Ballantyne A. Ethics of digital contact tracing wearables. J Med Ethics 2021. doi:10.1136/ medethics-2020-106958. [Epub ahead of print: 14 May 2021].

40 Kiang MV, Chin ET, Huynh BQ, et al. Routine asymptomatic testing strategies for airline travel during the COVID-19 pandemic: a simulation study. Lancet Infect Dis 2021;21:929-38.

41 World Health Organization. COVID-19 diagnostic testing in the context of international travel. Available: https://www.who.int/ publications/i/item/WHO-2019-nCoV-Sci_Brief-international_travel_ testing-2020.1

42 Singapore Ministry of Health. Maintaining vigilance with precautionary moves to better detect and reduce risk of local transmission of the omicron variant. Available: https://www.moh.gov sg/news-highlights/details/maintaining-vigilance-with-precautionarymoves-to-better-detect-and-reduce-risk-of-local-transmission-ofthe-omicron-variant

43 Hong Kong Government. Latest quarantine and testing arrangements for overseas arrivals and forthcoming implementation of self-paid serology antibody testing service at airport. Available: https://www.info.gov.hk/gia/general/202108/07/P2021080700596. htm?fontSize=1

44 Hong Kong Government. Airport serology antibody test shelved. Available: https://www.news.gov.hk/eng/2021/08/20210818/ 20210818 130917 492.html

45 Zhang Z, Bi Q, Fang S, et al. Insight into the practical performance of RT-PCR testing for SARS-CoV-2 using serological data: a cohort study. Lancet Microbe 2021;2:e79-87.

46 Hong Kong Government. Government further tightens testing and isolation arrangement for crew members of aircraft and other exempted persons. Available: https://www.info.gov.hk/gia/general/ 202011/21/P2020112100885.htm?fontSize=1

47 To KK-W, Chan W-M, Ip JD, KK-W T, JD I, et al. Unique clusters of severe acute respiratory syndrome coronavirus 2 causing a large coronavirus disease 2019 outbreak in Hong Kong. Clin Infect Dis 2021;73:137-42.

48 Hong Kong Government. Government announces tightening of testing and quarantine arrangement for exempted persons. Available: https://www.info.gov.hk/gia/general/202007/26/ P2020072600440.htm?fontSize $=1$
49 Singapore Civil Aviation Authority. CAAS to Further Tighten Measures to Ensure Safety and Well-Being of Air Crew. Available: https://www.caas.gov.sg/who-we-are/newsroom/Detail/caas-tofurther-tighten-measures-to-ensure-safety-and-well-being-of-aircrew/

50 Singapore Civil Aviation Authority. Update on measures in Changi Airport to safeguard public health. Available: https://www.caas.gov. sg/who-we-are/newsroom/Detail/update-on-measures-in-changiairport-to-safeguard-public-health/

51 Quilty BJ, Clifford S, Hellewell J, et al. Quarantine and testing strategies in contact tracing for SARS-CoV-2: a modelling study. Lancet Public Health 2021;6:e175-83.

52 Taiwan Ministry of Transportation and Communications. Supplementary of the principle of for flight crew epidemic prevention. Available: https://www.motc.gov.tw/ch/home.jsp?id=14\&parentpath= $0,2 \&$ mcustomize $=$ news_view.jsp\&dataserno $=202003270011 \&$

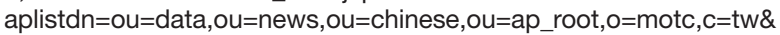
toolsflag $=Y$ \&imgfolder=img/standard\&fbclid=IwAR2FjismV1 fqYKCrbL u2OmJHdOeeSE-zmvmeY9LjnW5zEPI9QTX4_OEPO_g

53 Hong Kong Government. Cap. 599E compulsory quarantine of persons arriving at Hong Kong from foreign places regulation. Available: https://www.elegislation.gov.hk/hk/cap599E!en@2021-1029T00:00:00?INDEX CS=N\&xpid=ID 1584520336990 040

54 Singapore Ministry of Manpower. Four more work passes revoked for breaching Stay-Home notice requirements. Available: https:// www.mom.gov.sg/newsroom/press-releases/2020/1120-four-morework-passes-revoked-for-breaching-shn-requirements

55 Hong Kong Government. Man sentenced for breaching compulsory quarantine order. Available: https://www.info.gov.hk/gia/general/ 202112/17/P2021121700585.htm

56 Singapore Maritime and Port Authority. 47 individuals and 7 companies in the maritime sector Penalised for breaching safe management regulations. Available: https://www.mpa.gov.sg/web/ portal/home/media-centre/news-releases/detail/e0f90533-7da84c20-bc01-9ee522cdd54c

57 Hong Kong Government. Government invokes place-specific flight suspension mechanism. Available: https://www.info.gov.hk/gia/ general/202104/18/P2021041800858.htm?fontSize=1

58 Worsnop CZ. Concealing disease: trade and travel barriers and the timeliness of outbreak reporting. International Studies Perspectives 2019;20:344-72.

59 Cheng ZJ, Zhan Z, Xue M, et al. Public health measures and the control of COVID-19 in China. Clin Rev Allergy Immunol 2021;48:1-16.

60 Tan LF, Tan MF. Addressing endemic COVID-19 with high vaccination success: lessons from Singapore. J Am Med Dir Assoc 2021;22:2515-6.

61 Hong Kong Government. Measures for prevention and control of COVID-19 imposed by Guangdong Province on cross-boundary goods vehicle drivers entering Guangdong. Available: https:// www.info.gov.hk/gia/general/202007/27/P2020072700612.htm? fontSize $=1$ 